\title{
Carnets
}

Revue électronique d'études françaises de l'APEF

Première Série - 3 Numéro Spécial | 2011

La littérature face au "politiquement correct». Notions, pratiques et dérives

\section{La face (in)correcte du littéraire : considérations en guise d'avertissement}

José Domingues de Almeida

\section{(2) OpenEdition}

Journals

Édition électronique

URL : http://journals.openedition.org/carnets/6280

DOI : $10.4000 /$ carnets. 6280

ISSN : 1646-7698

Éditeur

APEF

Édition imprimée

Date de publication : 1 juin 2011

Pagination : 11-17

Référence électronique

José Domingues de Almeida, «La face (in)correcte du littéraire : considérations en guise

d'avertissement ", Carnets [En ligne], Première Série - 3 Numéro Spécial | 2011, mis en ligne le 19 juin

2018, consulté le 02 mai 2019. URL : http://journals.openedition.org/carnets/6280 ; DOI : 10.4000/ carnets. 6280

Carnets est mis à disposition selon les termes de la licence Creative Commons - Atribution - Pas d'utilisation commerciale 4.0 International. 


\section{LA FACE (IN)CORRECTE DU LITTERAIRE \\ Considérations en guise d'avertissement ${ }^{1}$}

José DOMINGUES DE ALMEIDA

FLUP - ILC Margarida Losa

jalmeida@letras.up.pt

\section{Résumé}

L'auteur propose une synthèse critique et sémantique de l'acception et de la pratique du "politiquement correct" en tant qu'idéologie appliquée subtilement à la littérature et dont il devine quelques dangers.

\section{Abstract}

The author proposes a critical and semantic synthesis of the meaning and practice of the "politically correct" ideology subtly applied to literature, and its dangers.

Mots-clés: littérature, politiquement correct, idéologie

Keywords: literature, politically correct, ideology

\footnotetext{
${ }^{1}$ Cette communication a été élaborée dans le cadre du projet "Interidentidades" de L'Institut de Literatura Comparada Margarida Losa de la Faculté des Lettres de l'Université de Porto, une I\&D subventionnée par la Fundação para a Ciência e a Tecnologia, intégrée dans le "Programa Operacional Ciência, Tecnologia e Inovação" (POCTI), Quadro de Apoio III (POCTI-SFA-18-500).
} 
La thématique du "politiquement correct", appliquée ou référée au fait littéraire peut sembler étrange, voire incongrue, au vu d'une approche de la littérature que a coutume de ne pas interroger le système dans lequel elle se meut, ni les retombées scripturales d'une idéologie sous-jacente, sournoise et potentiellement perverse, si l'on considère le propre de la création littéraire, notamment depuis la modernité. Mais, comme le rappelle judicieusement Christian Dufour: "Le politique se pense à la frontière du droit, de l'histoire et de la littérature - dans une certaine mesure aussi de la philosophie (...)" (Dufour, 2006: 13).

Né, comme bien des gadgets de la pensée et du dire, aux Etats-Unis, le concept de "politiquement correct" connaît chez nous un regain d'attention, d'acceptions et de mésusages ou d'abus depuis la fin des années quatre-vingt qui en fait un mot-valise, une bouée de sauvetage discursive et argumentaire quand le discours semble justement à bout d'arguments.

Le "politiquement correct", souvent rendu en français du Canada par "rectitude politique", entretient des accointances dangereuses avec des notions tout aussi floues et passe-partout, mais assumées par l'intelligentsia, comme "bienpensance" ou "pensée unique". Et pour cause!

Le fait est que, paradoxalement, nos régimes démocratiques ont implicitement consenti à ce que, moralement parlant, certaines choses ne se disent plus, que certaines expressions soient bannies du vocabulaire public, qu'un phénomène d'euphémisation généralisée se substitue à la diction des différences, des discordances et des négativités.

Nous vivons, en effet, - société médiatisée et un certain journalisme aidant -, dans le monde du langage surveillé, du discours qui ne blesse pas, de l'innocuité langagière et de la langue de bois comme horizons et moyens du discours. Faut-il s'en inquiéter? Faut-il craindre une perte de négativité de la littérature comme discours opposé à la langue de la communication et de la correspondance sociale?

Certains théoriciens semblent le penser qui rejoignent çà et là quelques écrivains qui osent encore braver les interdits du moment, les conventions inoffensives du discours ambiant au profit d'une parole de dissension ou de différend.

Pour la fiction narrative, une tradition de l'écriture du négatif ou de rupture par rapport à l'écriture de confort et de conformité sociale, qui va de Rabelais jusqu'à Denis Roche, en passant par Sade ou Lautréamont, mais qui connaît encore des émules dans la fiction narrative française contemporaine comme Eugène Savitzkaya, Valère Novarina, Eric Chevillard, Richard Millet ou Michel Houellebecq, comme naguère Pierre Guyotat, - "ceux qui merdrent", pour reprendre Christian Prigent -, assure une veille éthique de l'écriture, et la sauvegarde par rapport au risque toujours menaçant d'une transparence et d'une évidence du message littéraire, trop souvent livré au goût du jour (Prigent,1991: 11-29). 
Prigent rappelle cette exigence éthique et esthétique placée au centre même de la dynamique moderne de la représentation: "Le surgissement du moderne, c'était donc le dénudement des conflits, la prise de parti dans ces conflits, la capacité, pour le langage littéraire, de prendre en charge ces conflits, de leur donner forme dans des écritures travaillées par leur violence" (idem: 23); raison pour laquelle cet auteur regrette et s'insurge contre la défaite de la pensée qui renonce à "affronter ce que Kafka appelait le négatif, Bataille, la part maudite et Artaud la cruauté" (idem: 25s).

Même constat angoissé pour la poésie contemporaine chez $\mathrm{J} .-\mathrm{Cl}$. Pinson pour qui “(...) toute vraie poésie est dissidence au regard des langages eux-mêmes préfabriqués dont nous sommes de plus en plus envahis (de cette langue molle et relâchée, 'muesli' (...) qui gangrène le parler quotidien, à ces langues cybernétiques qui prolifèrent sur les écrans). Dissidence aussi à l'encontre de tous les 'poétismes'” (Pinson, 1999: 41).

Étrange paradoxe que celui où nous nous trouvons et qui justifie cette réflexion. Comment, alors que nous vivons et écrivons sous un régime politique fondé sur la plus large liberté d'expression qu'il soit, se soumettre à une logique d'évitement du signifiant, de courtcircuitage du sens ou d'adhésion consciente ou inconsciente à une pensée unique qui, tout en ne heurtant personne, n'en fait pas moins patiner la fiction et la parole poétique dans un programme discursif auquel elles seraient censées se plier?

Des voix se font entendre, il est vrai, qui contestent cette idéologie du "ne pas dire" et du "ne pas écrire", de la surveillance du discours et du consensus mou ou mièvre. De même, plusieurs dictionnaires du "politiquement correct" (Cf. Kahn, 2005) ont vu le jour qui illustrent et désavouent une sorte de ravissement du signe linguistique et le principe assumé d'une euphémisation obligatoire de la pensée, du discours, voire de la politique.

Pour ce qui est des textes narratifs, une certaine mouvance de la fiction romanesque contemporaine en français se fait en effet l'écho d'un même souci: pouvoir appeler les choses, les phénomènes et les gens par leurs noms; s'insurger contre une idéologie discursive consensuelle, souvent colportée par un journalisme bon marché, qui empêche d'envisager les faits sociaux, politiques et culturels sous un jour complexe et décomplexé; qui évitent d'approcher l'héritage historique sans craindre un devoir de repentance personnelle et ethnique ${ }^{2}$; concevoir une convivialité sociétale affranchie d'une logique de quotas et de minorisation des compétences, des savoirs et des pouvoirs; c'est-à-dire de prétextes et d'alibis sociaux. Cette même mouvance met parfois subtilement en scène, comme c'est le cas chez Michel Houellebecq, un désaveu grinçant des retombées politiques et civilisationnelles de l'application des modèles de gestions sociétales des différences,

\footnotetext{
${ }^{2}$ On se rappellera que, par exemple, l'argumentaire politique de la campagne présidentielle de Nicolas Sarkozy a intensément joué la carte du refus de la "repentance“, notamment de la relecture contrite de l'Histoire récente, notamment du fait colonial.
} 
notamment le recours au concept de "discrimination positive", lequel met à mal des décennies d'idéals d'intégration à la française (Cf. Schnapper, 2007 \& Cf. Doytcheva, 2005: 23-30).

Cette approche n'est certes pas sans conséquences pour la culture telle que nous la concevons, ou sommes en passe de la concevoir çà et là. Paradoxe, à nouveau, c'est au nom des "French Theory" ou "French Studies", à la matrice si peu "hexagonale", que l'on a fomenté une dissémination, voire une ghettoïsation de la culture aux Etats-Unis, corolaire universitaire et scientifique d'une idéologie "politiquement correcte" où s'entassent des études féminines, sexuelles, homosexuelles, postcoloniales, lesbiennes, noires et tant d'autres... Autant d'embrasures attrayantes, il est vrai, d'un édifice culturel que l'on refuse ou évite d'intégrer par la grande porte.

D'aucuns n'hésitent même pas à mettre les Etudes Francophones dans ce sac culturel ambivalent et interrogent l'(in)aptitude hexagonale à mettre en perspective les écritures francophones périphériques selon ce prisme d'approche; ce qui reviendrait à considérer ce champ comme l'expression d'une manifestation littéraire minorisée, protégée ou tolérée quelque part par la pensée correcte bienveillante et inoffensive ( $C f$. Baetens, 2003: 39-47).

En fait, la pensée "politiquement correct", si jamais elle existe en tant que telle et qu'elle dégage un discours sociopolitique cohérent, pointe aussi une difficulté à interroger les postures et les fondements idéologiques des pratiques pédagogiques et de l'éducation en général. On ne s'étonnera pas de voir plusieurs essais sur l'échec des politiques éducatives, basées sur le mythe du bon sauvage et des approches tautologiques et circulaires infinies de l'apprentissage: "apprendre à apprendre à apprendre à..." (le "pédagogiquement correct") ou sur la déculpabilisation d'office de l'apprenant ou l'évitement facilitiste de l'effort mémoriel, et de l'effort tout court, provoquer les scandales, ou les remises en cause que l'on sait (Cf. Coignard, 2011) ${ }^{3}$.

On l'aura compris aussi: tout un discours décliniste hexagonal, et étranger sur le devenir de la culture française qui, depuis quelque vingt ans, vitupère contre l'état des lieux culturel de la France, mais qui touche aussi à l'organisation politique et sociale de ce pays, est sporadiquement en phase avec cette méfiance à l'égard d'une rectitude politique et culturelle, donc aussi "littéraire" quelque part, qui entrave une pensée différente, et ankylose le débat de société (Cf. Morrison, 2008 \& Compagnon, 2008).

La littérature peut-elle encore être le lieu de cette dissidence par rapport à la pensée unique et à la communication, voire la "com"”, comme les médias aiment à la désigner aujourd'hui pour mieux l'évider? Est-elle encore à même de choquer alors que tout semble

\footnotetext{
${ }^{3}$ L'idéologie bienpensante dans le système éducatif chez nous, Portugais, a pour nom "eduquês"; cette langue de bois programmatique dont s'entichent des "spécialistes" et qui produit thèses et colloques à l'envi.
} 
avoir été écrit et lu? Quelle est sa marge de manœuvre, et quelle peut encore être la morale du littéraire?

Plusieurs écrivains français et francophones contemporains s'inscrivent en faux par rapport à cette rectitude politique et n'hésitent pas à remuer le couteau fictionnel dans la plaie discursive du moment. Aussi bien par le style que par la thématique, surtout, ils sont plusieurs à complexifier le débat et à enrichir la polémique sociétale. N'en déplaise à d'autres qui font plutôt dans le douillet et le calfeutré... Mais qui vendent bien également ! II n'est pas que le scandale pour produire un scoop. Rappelons-nous Delerm ou Bobin pour faire court!

Ainsi, Richard Millet revendique fièrement sa francité. Est-ce un mal? Houellebecq nous renvoie souvent à la figure nos incohérences dans la gestion du modèle d'intégration et les ambigüités des générations formées dans la foulée de la pensée 68. Faut-il pour autant le mettre au ban de l'établissement littéraire alors que, belle reconnaissance, le Prix Goncourt vient de lui être décerné? De même pour Philippe Murray.

Par ailleurs, l'écriture narrative de Christine Angot ou d'Amélie Nothomb conçoit, tout comme d'autres écrivains du moment, Dieu merci, la pratique de l'écriture littéraire comme une provocation et indignation permanentes et subtiles; ce qu'une certaine taxinomie de la contemporanéité fictionnelle française a fort bien nommé "la tradition de l'insolence" et que Dominique Viart attribue au moment spécifique vécu par nos sociétés; à savoir un "désenchantement cynique et provocateur, parfois drôle dans la neutralité factuelle de ses constats et les 'court-circuits' de l'écriture" (Cf. Viart, 1996: 103).

N'est-ce pas cette capacité d'étonner et de provoquer que l'on attend toujours nostalgiquement et paradoxalement, surtout à l'étranger, quelque part des Français et de la France, comme le rappelle Perry Anderson:

L'idéologie des droits de l'homme, toute libérale qu'elle parût - n'avait-elle pas été le plat de résistance du banquet idéologique du Bicentenaire? -, ne constituait pas une politique. Succédané contemporain de ce qui avait été autrefois les idéaux du socialisme, elle sapait la cohérence de la notion en tant qu'être collectif, et elle aboutissait à des exigences intrinsèquement contradictoires: droit à l'égalité et droit à la différence proclamés dans un même souffle (Anderson, 2005: 59).

Dans le même sens, plusieurs théoriciens de la géopolitique et géolinguistique française en contexte mondialisé n'ont de cesse de rappeler une spécificité francophone qui abhorre les consensus et qui pointe une "civilisation de langue française" que décrit tant par la diachronie que par l'histoire toute récente un Christian Dufour: 
L'existence d'une vision francophone du monde à prétention universaliste assure un minimum de légitimité aux autres mouvements d'opposition, qu'un début de nouvel ordre international sous la houlette américaine associe automatiquement au terrorisme, ou aux aberrations bureaucratiques de l'ancien bloc soviétique (Dufour, 2006: 21).

La question est dès lors de savoir si cette tradition de l'insolence se dote du pouvoir de frapper au-delà des effets médiatiques et commerciaux, et si elle entend assurer une veille éthique de l'écriture dans le contexte contemporain; ce qui assignerait à la pratique littéraire, écriture et lecture confondues, une fonction incontournable dans la Cité globale. 


\section{Bibliographie}

ANDERSON, Perry (2005). La pensée tiède. Un regard critique sur la culture française. Paris: Seuil.

BAETENS, Jan (2003). "Les 'études culturelles', encore une exception française?”. In: L. D'Hulst et J-M. Moura (orgs). Les études littéraires francophones: état des lieux. Lille: Un. Lille 3, pp. 39-47.

CoIGNARD, Sophie (2011). Le pacte immoral. Paris: Albin Michel.

Compagnon, Antoine (2008). Le souci de la grandeur. Paris: Denoël.

DOYTCHEVA, Milena (2005). Le multiculturalisme. Paris: La découverte.

DuFOUR, Christian (2006). Le défi français. Regards croisés sur la France et le Québec. Montréal: Septentrion.

KHAN, Jean-François (2005). Dictionnaire incorrect. Paris: Plon.

MORRISON, David (2008). Que reste-t-il de la culture française?. Paris: Denoël.

PINSON, Jean-Claude (1999). A quoi bon la poésie aujourd'hui?. Paris: Ed. Pleins Feux.

Prigent, Christian (1991). Ceux qui merdRent. Paris: P.O.L

SCHNAPPER, Dominique (2007). Qu'est-ce que l'intégration?. Paris: Gallimard.

VIART, Dominique (1996). "Le roman en question III". In: Prétexte, no 11, pp. 63-68. 
